\title{
EXISTENCE AND UNIFORM DECAY FOR A NONLINEAR BEAM EQUATION WITH NONLINEARITY OF KIRCHHOFF TYPE IN DOMAINS WITH MOVING BOUNDARY
}

\author{
M. L. SANTOS, J. FERREIRA, AND C. A. RAPOSO
}

Received 2 October 2003

We prove the exponential decay in the case $n>2$, as time goes to infinity, of regular solutions for the nonlinear beam equation with memory and weak damping $u_{t t}+\Delta^{2} u-$ $M\left(\|\nabla u\|_{L^{2}\left(\Omega_{t}\right)}^{2}\right) \Delta u+\int_{0}^{t} g(t-s) \Delta u(s) d s+\alpha u_{t}=0$ in $\hat{Q}$ in a noncylindrical domain of $\mathbb{R}^{n+1}(n \geq 1)$ under suitable hypothesis on the scalar functions $M$ and $g$, and where $\alpha$ is a positive constant. We establish existence and uniqueness of regular solutions for any $n \geq 1$.

\section{Introduction}

Let $\Omega$ be an open bounded domain of $\mathbb{R}^{n}$ containing the origin and having $C^{2}$ boundary. Let $\gamma:[0, \infty[\rightarrow \mathbb{R}$ be a continuously differentiable function. See hypotheses (1.24), (1.25), and (1.26) on $\gamma$. Consider the family of subdomains $\left\{\Omega_{t}\right\}_{0 \leq t<\infty}$ of $\mathbb{R}^{n}$ given by

$$
\Omega_{t}=T(\Omega), \quad T: y \in \Omega \longmapsto x=\gamma(t) y,
$$

whose boundaries are denoted by $\Gamma_{t}$, and let $\hat{Q}$ be the noncylindrical domain of $\mathbb{R}^{n+1}$ given by

$$
\hat{Q}=\bigcup_{0 \leq t<\infty} \Omega_{t} \times\{t\}
$$

with lateral boundary

$$
\hat{\Sigma}=\bigcup_{0 \leq t<\infty} \Gamma_{t} \times\{t\} .
$$

We consider the Hilbert space $L^{2}(\Omega)$ endowed with the inner product

$$
(u, v)=\int_{\Omega} u(x) v(x) d x
$$


and corresponding norm

$$
\|u\|_{L^{2}(\Omega)}^{2}=(u, u) .
$$

We also consider the Sobolev space $H^{1}(\Omega)$ endowed with the scalar product

$$
(u, v)_{H^{1}(\Omega)}=(u, v)+(\nabla u, \nabla v) .
$$

We define the subspace of $H^{1}(\Omega)$, denoted by $H_{0}^{1}(\Omega)$, as the closure of $C_{0}^{\infty}(\Omega)$ in the strong topology of $H^{1}(\Omega)$. By $H^{-1}(\Omega)$, we denote the dual space of $H_{0}^{1}(\Omega)$. This space endowed with the norm induced by the scalar product

$$
((u, v))_{H_{0}^{1}(\Omega)}=(\nabla u, \nabla v)
$$

is, owing to the Poincaré inequality

$$
\|u\|_{L^{2}(\Omega)}^{2} \leq C\|\nabla u\|_{L^{2}(\Omega)}^{2},
$$

a Hilbert space. We define for all $1 \leq p<\infty$,

$$
\|u\|_{L^{p}(\Omega)}^{p}=\int_{\Omega}|u(x)|^{p} d x,
$$

and if $p=\infty$,

$$
\|u\|_{L^{\infty}(\Omega)}=\sup _{x \in \Omega} \operatorname{ess}|u(x)| .
$$

In this work, we study the existence and uniqueness of strong solutions as well as the exponential decay of the energy to the nonlinear beam equation with memory given by

$$
\begin{gathered}
u_{t t}+\Delta^{2} u-M\left(\|\nabla u\|_{L^{2}\left(\Omega_{t}\right)}^{2}\right) \Delta u+\int_{0}^{t} g(t-s) \Delta u(s) d s+\alpha u_{t}=0 \quad \text { in } \hat{Q}, \\
u=\frac{\partial u}{\partial \nu}=0 \quad \text { on } \sum \\
u(x, 0)=u_{0}(x), \quad u_{t}(x, 0)=u_{1}(x) \quad \text { in } \Omega_{0},
\end{gathered}
$$

where $\nu=\nu(\sigma, t)$ is the unit normal at $(\sigma, t) \in \hat{\sum}$ directed towards the exterior of $\hat{Q}$. If we denote by $\eta$ the outer unit normal to the boundary $\Gamma$ of $\Omega$, we have, using a parametrization of $\Gamma$,

$$
\nu(\sigma, t)=\frac{1}{\nu}\left(\eta(\xi),-\gamma^{\prime}(t) \xi \cdot \eta(\xi)\right), \quad \xi=\frac{\sigma}{\gamma(t)},
$$

where

$$
\nu=\left(1+\gamma^{\prime}(t)|\xi \cdot \eta(\xi)|^{2}\right)^{1 / 2}
$$


Indeed, fix $(\sigma, t) \in \hat{\sum}$. Let $\varphi=0$ be a parametrization of a part $U$ of $\Gamma, \cup$ containing $\xi=\sigma / \gamma(t)$. The parametrization of a part $\bigcup$ of $\hat{\sum}$ is $\psi(\sigma, t)=\varphi(\sigma / \gamma(t))=\varphi(\xi)=0$. We have

$$
\nabla \psi(\sigma, t)=\frac{1}{\gamma(t)}\left(\nabla \varphi(\xi),-\gamma^{\prime}(t) \xi \cdot \nabla \varphi(\xi)\right)
$$

From this and observing that $\eta(\xi)=\nabla \varphi(\xi) /|\nabla \varphi(\xi)|$, expression (1.14) follows. Let $\bar{\nu}(\cdot, t)$ be the $x$-component of unit normal $\nu(\cdot, \cdot),|\bar{\nu}| \leq 1$. Then by relation (1.14), one has

$$
\bar{\nu}(\sigma, t)=\eta\left(\frac{\sigma}{\gamma(t)}\right)
$$

In this paper, we deal with the nonlinear beam equation with memory in domains with moving boundary. We show the existence and uniqueness of strong solutions to the initial boundary value problem (1.11)-(1.13). The method we use to prove the result of existence and uniqueness is based on transforming our problem into another initial boundary value problem defined over a cylindrical domain whose sections are not time dependent. This is done using a suitable change of variable. Then we show the existence and uniqueness for this new problem. Our existence result on domains with moving boundary will follow by using the inverse transformation, that is, by using the diffeomorfism

$$
\tau: \hat{Q} \longrightarrow Q, \quad(x, t) \in \Omega_{t} \longmapsto(y, t)=\left(\frac{x}{\gamma(t)}, t\right)
$$

and $\tau^{-1}: Q \rightarrow \hat{Q}$ defined by

$$
\tau^{-1}(y, t)=(x, t)=(\gamma(t) y, t) .
$$

Denoting by $v$ the function

$$
v(y, t)=u \circ \tau^{-1}(y, t)=u(\gamma(t) y, t),
$$

the initial boundary value problem (1.11)-(1.13) becomes

$$
\begin{gathered}
v_{t t}+\gamma^{-4} \Delta^{2} v-\gamma^{-2} M\left(\gamma^{n-2}\|\nabla v\|_{L^{2}(\Omega)}^{2}\right) \Delta v+\int_{0}^{t} g(t-s) \gamma^{-2}(s) \Delta v(s) d s \\
+\alpha v_{t}-A(t) v+a_{1} \cdot \nabla \partial_{t} v+a_{2} \cdot \nabla v=0 \quad \text { in } Q, \\
\left.v\right|_{\Gamma}=\left.\frac{\partial v}{\partial v}\right|_{\Gamma}=0, \\
\left.v\right|_{t=0}=v_{0},\left.\quad v_{t}\right|_{t=0}=v_{1} \quad \text { in } \Omega,
\end{gathered}
$$


where

$$
\begin{gathered}
A(t) v=\sum_{i, j=1}^{n} \partial_{y_{i}}\left(a_{i j} \partial_{y_{j}} v\right), \\
a_{i j}(y, t)=-\left(\gamma^{\prime} \gamma^{-1}\right)^{2} y_{i} y_{j} \quad(i, j=1, \ldots, n) \\
a_{1}(y, t)=-\gamma^{\prime} \gamma^{-1} y, \\
a_{2}(y, t)=-\gamma^{-2} y\left(\gamma^{\prime \prime} \gamma+\gamma^{\prime}\left(\alpha \gamma+(n-1) \gamma^{\prime}\right)\right) .
\end{gathered}
$$

To show the existence of strong solution, we will use the following hypotheses:

$$
\begin{gathered}
\gamma^{\prime} \leq 0 \quad n>2, \quad \gamma^{\prime} \geq 0 \quad \text { if } n \leq 2, \\
\gamma \in L^{\infty}(0, \infty), \quad \inf _{0 \leq t<\infty} \gamma(t)=\gamma_{0}>0, \\
\gamma^{\prime} \in W^{2, \infty}(0, \infty) \cap W^{2,1}(0, \infty) .
\end{gathered}
$$

Note that assumption (1.24) means that $\hat{Q}$ is decreasing if $n>2$ and increasing if $n \leq 2$ in the sense that when $t>t^{\prime}$ and $n>2$, then the projection of $\Omega_{t^{\prime}}$ on the subspace $t=$ 0 contains the projection of $\Omega_{t}$ on the same subspace and contrary in the case $n \leq 2$. The above method was introduced by Dal Passo and Ughi [4] to study certain class of parabolic equations in noncylindrical domains. Concerning the function $M \in C^{1}[0, \infty$, we assume that

$$
M(\tau) \geq-m_{0}, \quad M(\tau) \tau \geq \hat{M}(\tau) \quad \forall \tau \geq 0,
$$

where $\hat{M}(\tau)=\int_{0}^{\tau} M(s) d s$ and

$$
0 \leq m_{0}<\lambda_{1}\|\gamma\|_{L^{\infty}}^{-2}
$$

where $\lambda_{1}$ is the first eigenvalue of the spectral Dirichlet problem

$$
\Delta^{2} w=\lambda_{1} w \quad \text { in } \Omega, \quad w=\frac{\partial w}{\partial \eta}=0 \quad \text { in } \Gamma .
$$

We recall also the classical inequality

$$
\|\Delta w\|_{L^{2}(\Omega)} \geq \sqrt{\lambda_{1}}\|\nabla w\|_{L^{2}(\Omega)} .
$$

Remark 1.1. The hypotheses (1.27) and (1.30) are classic, as one can see, for instance, in $[9,20,21]$ without the term of memory $\int_{0}^{t} g(t-s) \Delta u(s) d s$ in fixed domain. In fact, the hypothesis (1.28) was introduced by the second author with some modifications, due to the complexity of working in noncylindrical domains in [1].

Unlike the existing papers on stability for hyperbolic equations in noncylindrical domain, we do not use the penalty method introduced by Lions [16], but work directly in our noncylindrical domain $\hat{Q}$. To see the dissipative properties of the system, we have to construct a suitable functional whose derivative is negative and is equivalent to the 
first-order energy. This functional is obtained using the multiplicative technique following Komornik [10] or Rivera [18]. We only obtained the exponential decay of solution for our problem for the case $n>2$. The main difficult to obtain the decay for $n \leq 2$ is due to the geometry of the noncylindrical domain because it affects substantially the problem, since we work directly in $\hat{Q}$. Therefore the case $n \leq 2$ is an important open problem. From the physics point of view, the system (1.11)-(1.13) describes the transverse deflection of a streched viscoelastic beam fixed in a moving boundary device. The viscoelasticity property of the material is characterized by the memory term

$$
\int_{0}^{t} g(t-s) \Delta u(s) d s
$$

The uniform stabilization of plates equations with linear or nonlinear boundary feedback was investigated by several authors, see for example $[8,9,11,13,14,15]$. In a fixed domain, it is well known that if the relaxation function $g$ decays to zero, then the energy of the system also decays to zero, see $[3,12,19,22]$. But in a moving domain, the transverse deflection $u(x, t)$ of a beam which changes its configuration at each instant of time increases its deformation, and hence increases its tension. Moreover, the horizontal movement of the boundary yields nonlinear terms involving derivatives in the space variable. To control these nonlinearities, we add in the system a frictional damping, characterized by $u_{t}$. This term will play an important role in the dissipative nature of the problem. In $[1,6]$, a quite complete discussion about the model of transverse deflection and transverse vibrations can be found, respectively, for the nonlinear beam equation and elastic membranes. This model was proposed by Woinowsky-Krieger [23] for the case of cylindrical domains, without the dissipative term and $\int_{0}^{t} g(t-s) \Delta u(s) d s$. See also Eisley [5] and Burgreen [2] for physics justification and background of the model. Our results in this paper were more difficult to obtain than the results in [7], due to the introduction of the terms corresponding to the biharmonic operator $\Delta^{2}$ and to the nonlinear function of Kirchhoff type $M\left(\|\nabla u\|_{L^{2}(\Omega)}^{2}\right)$, which generated nontrivial problems that were solved thanks to the hypotheses (1.27), (1.28), and (1.30) and to the hypothesis regarding the "dilation function". Besides, in [7], we made only two estimates, while here we had to make four estimates that introduce some technical ideas with regard to the existence, uniqueness, and regularity. Regarding the solution decay, we used a similar technical of [7] but we introduced Lemmas 3.3 and 3.4 to control the terms of energy and to use with success the technique of multipliers. We use the standard notations which can be found in Lion's and Magenes' books $[16,17]$. In the sequel by $C$ (sometimes $C_{1}, C_{2}, \ldots$ ), we denote various positive constants which do not depend on $t$ or on the initial data. This paper is organized as follows. In Section 2, we prove a basic result on existence, regularity, and uniqueness of regular solutions. We use Galerkin approximation, Aubin-Lions theorem, energy method introduced by Lions [16], and some technical ideas to show existence regularity and uniqueness of regular solution for problem (1.11)-(1.13). Finally, in Section 3, we establish a result on the exponential decay of the regular solution to the problem (1.11)-(1.13). We use the technique of the multipliers introduced by Komornik [10], Lions [16], and Rivera [18] coupled with some technical lemmas and some technical ideas. 


\section{Existence and regularity}

In this section, we will study the existence and regularity of solutions for the system (1.11)-(1.13). For this, we assume that the kernel $g: \mathbb{R}_{+} \rightarrow \mathbb{R}_{+}$is in $W^{2,1}(0, \infty)$, and satisfies

$$
g,-g^{\prime} \geq 0, \quad \frac{m_{1}}{\|\gamma\|_{L^{\infty}}^{2}}-\int_{0}^{\infty} g(s) \gamma^{-2}(s) d s=\beta_{1}>0,
$$

where

$$
m_{1}=\left(\frac{\lambda_{1}}{\|\gamma\|_{L^{\infty}}^{2}}-m_{0}\right)>0 .
$$

To simplify our analysis, we define the binary operator

$$
g \square \frac{\varphi(t)}{\gamma(t)}=\int_{\Omega} \int_{0}^{t} g(t-s) \gamma^{-2}(s)|\varphi(t)-\varphi(s)|^{2} d s d x .
$$

With this notation, we have the following statement.

Lemma 2.1. For $v \in C^{1}\left(0, T: H_{0}^{2}(\Omega)\right)$,

$$
\begin{aligned}
& \int_{\Omega} \int_{0}^{t} g(t-s) \gamma^{-2}(s) \nabla v(s) \cdot \nabla v_{t}(t) d s d x \\
& \quad=-\frac{1}{2} \frac{g(t)}{\gamma^{2}(0)} \int_{\Omega}|\nabla v|^{2} d x+\frac{1}{2} g^{\prime} \square \frac{\nabla v}{\gamma}-\frac{1}{2} \frac{d}{d t}\left[g \square \frac{\nabla v}{\gamma}-\left(\int_{0}^{t} \frac{g(s)}{\gamma^{2}(s)} d s\right) \int_{\Omega}|\nabla v|^{2} d x\right], \\
& \int_{\Omega} \int_{0}^{t} g(t-s) \gamma^{-2}(s) \Delta v \Delta v_{t} d s d x \\
& \quad=-\frac{1}{2} \frac{g(t)}{\gamma^{2}(0)} \int_{\Omega}|\Delta v|^{2} d x+\frac{1}{2} g^{\prime} \square \frac{\Delta v}{\gamma}-\frac{1}{2} \frac{d}{d t}\left[g \square \frac{\Delta v}{\gamma}-\left(\int_{0}^{t} \frac{g(s)}{\gamma^{2}(s)} d s\right) \int_{\Omega}|\Delta v|^{2} d x\right] .
\end{aligned}
$$

The proof of this lemma follows by differentiating the terms $g \square(\nabla u(t) / \gamma(t))$ and $g \square(\Delta u(t) / \gamma(t))$. The well posedness of system (1.21) is given by the following theorem.

Theorem 2.2. Take $v_{0} \in H_{0}^{2}(\Omega) \cap H^{4}(\Omega), v_{1} \in H_{0}^{2}(\Omega)$, and suppose that assumptions (1.24), (1.25), (1.26), (1.27), (1.28), (1.30), and (2.1) hold. Then there exists a unique solution $v$ of the problem (1.21) satisfying

$$
\begin{gathered}
v \in L^{\infty}\left(0, \infty: H_{0}^{2}(\Omega) \cap H^{4}(\Omega)\right), \\
v_{t} \in L^{\infty}\left(0, \infty: H_{0}^{1}(\Omega)\right), \\
v_{t t} \in L^{\infty}\left(0, \infty: L^{2}(\Omega)\right) .
\end{gathered}
$$


Proof. We denote by $B$ the operator

$$
B w=\Delta^{2} w, \quad D(B)=H_{0}^{2}(\Omega) \cap H^{4}(\Omega) .
$$

It is well known that $B$ is a positive selfadjoint operator in the Hilbert space $L^{2}(\Omega)$ for which there exist sequences $\left\{w_{n}\right\}_{n \in \mathbb{N}}$ and $\left\{\lambda_{n}\right\}_{n \in \mathbb{N}}$ of eigenfunctions and eigenvalues of $B$ such that the set of linear combinations of $\left\{w_{n}\right\}_{n \in \mathbb{N}}$ is dense in $D(B)$ and $\lambda_{1}<\lambda_{2} \leq \cdots \leq$ $\lambda_{n} \rightarrow \infty$ as $n \rightarrow \infty$. We denote by

$$
v_{0}^{m}=\sum_{j=1}^{m}\left(v_{0}, w_{j}\right) w_{j}, \quad v_{1}^{m}=\sum_{j=1}^{m}\left(v_{1}, w_{j}\right) w_{j} .
$$

Note that for any $\left(v_{0}, v_{1}\right) \in D(B) \times H_{0}^{2}(\Omega)$, we have $v_{0}^{m} \rightarrow v_{0}$ strong in $D(B)$ and $v_{1}^{m} \rightarrow v_{1}$ strong in $H_{0}^{2}(\Omega)$.

We denote by $V_{m}$ the space generated by $w_{1}, \ldots, w_{m}$. Standard results on ordinary differential equations imply the existence of a local solution $v^{m}$ of the form

$$
v^{m}(t)=\sum_{j=1}^{m} g_{j m}(t) w_{j},
$$

to the system

$$
\begin{gathered}
\int_{\Omega} v_{t t}^{m} w_{j} d y+\alpha \int_{\Omega} v_{t}^{m} w_{j} d y+\int_{\Omega} \gamma^{-4} \Delta^{2} v^{m} w_{j} d y \\
-\gamma^{-2} M\left(\gamma^{n-2}\left\|\nabla v^{m}\right\|_{L^{2}(\Omega)}^{2}\right) \int_{\Omega} \Delta v^{m} w_{j} d y \\
+\int_{\Omega} \int_{0}^{t} g(t-s) \gamma^{-2}(s) \nabla v^{m}(s) d s \cdot \nabla w_{j} d y+\int_{\Omega} A(t) v^{m} w_{j} d y \\
+\int_{\Omega} a_{1} \cdot \nabla v_{t}^{m} w_{j} d y+\int_{\Omega} a_{2} \cdot \nabla v^{m} w_{j} d y=0, \quad(j=1, \ldots, m) \\
v^{m}(x, 0)=v_{0}^{m}, \quad v_{t}^{m}(x, 0)=v_{1}^{m} .
\end{gathered}
$$

The extension of these solutions to the interval $[0, \infty$ [ is a consequence of the first estimate which we are going to prove below.

A priori estimate I. Multiplying (2.9) by $g_{j m}^{\prime}(t)$, summing up the resulting product in $j=1,2, \ldots, m$, and after some calculations using Lemma 2.1 , we get

$$
\begin{aligned}
\frac{1}{2} \frac{d}{d t} \mathfrak{E}_{1}^{m}( & \left.t, v^{m}\right)+\alpha\left\|v_{t}^{m}\right\|_{L^{2}(\Omega)}^{2}-\frac{(n-2) \gamma^{\prime}}{2 \gamma^{n+1}} \\
& \times\left[\gamma^{n-2}\left\|\nabla v^{m}\right\|_{2}^{2} M\left(\gamma^{n-2}\left\|\nabla v^{m}\right\|_{L^{2}(\Omega)}^{2}\right)-\hat{M}\left(\gamma^{n-2}\left\|\nabla v^{m}\right\|_{L^{2}(\Omega)}^{2}\right)\right] \\
& +\int_{\Omega} A(t) v^{m} v_{t}^{m} d y+\int_{\Omega} a_{1} \cdot \nabla v_{t}^{m} v_{t}^{m} d y+\int_{\Omega} a_{2} \cdot \nabla v^{m} v_{t}^{m} d y \\
= & -\frac{1}{2} \frac{g(t)}{\gamma^{2}(0)}\left\|\nabla v^{m}\right\|_{L^{2}(\Omega)}^{2}+\frac{1}{2} g^{\prime} \square \frac{\nabla v^{m}}{\gamma}-4 \frac{\gamma^{\prime}}{\gamma^{5}}\left\|\Delta v^{m}\right\|_{L^{2}(\Omega)}^{2},
\end{aligned}
$$


where

$$
\begin{aligned}
\mathfrak{E}_{1}^{m}\left(t, v^{m}\right)= & \left\|v_{t}^{m}\right\|_{L^{2}(\Omega)}^{2}+\left(-\int_{0}^{t} g(s) \gamma^{-2}(s) d s\right)\left\|\nabla v^{m}\right\|_{L^{2}(\Omega)}^{2} \\
& +\gamma^{-4}\left\|\Delta v^{m}\right\|_{L^{2}(\Omega)}^{2}+\gamma^{-n} \hat{M}\left(\gamma^{n-2}\left\|\nabla v^{m}\right\|_{L^{2}(\Omega)}^{2}\right)+g \square \frac{\nabla v^{m}}{\gamma} .
\end{aligned}
$$

From (1.27), (1.28), and (1.30), it follows that

$$
\gamma^{-4}\left\|\Delta v^{m}\right\|_{L^{2}(\Omega)}^{2}+\gamma^{-n} \hat{M}\left(\gamma^{n-2}\left\|\nabla v^{m}\right\|_{L^{2}(\Omega)}^{2}\right) \geq \frac{m_{1}}{\|\gamma\|_{L^{\infty}}^{2}}\left\|\nabla v^{m}\right\|_{L^{2}(\Omega)}^{2} .
$$

Taking into account (1.24), (1.27), the last inequality, and (2.1), it follows that the equality (2.11) can be written as

$$
\frac{1}{2} \frac{d}{d t} \mathfrak{E}_{1}^{m}\left(t, v^{m}\right)+\alpha\left\|v_{t}^{m}\right\|_{L^{2}(\Omega)}^{2} \leq C\left(\left|\gamma^{\prime}\right|+\left|\gamma^{\prime \prime}\right|\right) \mathfrak{E}_{1}^{m}(t) .
$$

Integrating the inequality (2.14), using Gronwall's lemma, and taking into account (1.26), we get

$$
\mathfrak{E}_{1}^{m}\left(t, v^{m}\right)+\int_{0}^{t}\left\|v_{s}^{m}(s)\right\|_{L^{2}(\Omega)}^{2} d s \leq C, \quad \forall m \in \mathbb{N}, \forall t \in[0, T]
$$

A priori estimate II. Now, if we multiply (2.9) by $\sqrt{\lambda_{j}} g_{j m}^{\prime}(t)$ and summing up in $j=$ $1, \ldots, m$, we get after some calculations

$$
\begin{aligned}
\frac{1}{2} \frac{d}{d t} \| & \nabla v_{t}^{m}\left\|_{L^{2}(\Omega)}^{2}+\alpha\right\| \nabla v_{t}^{m}\left\|_{L^{2}(\Omega)}^{2}+\frac{\gamma^{-2}}{2} M\left(\gamma^{n-2}\left\|\nabla v^{m}\right\|_{L^{2}(\Omega)}^{2}\right) \frac{d}{d t}\right\| \Delta v^{m} \|_{L^{2}(\Omega)}^{2} \\
& +\frac{\gamma^{-4}}{2} \frac{d}{d t}\left\|\nabla \Delta v^{m}\right\|_{L^{2}(\Omega)}^{2}-\int_{\Omega} \int_{0}^{t} g(t-s) \gamma^{-2}(s) \Delta v^{m}(s) \Delta v_{t}^{m} d s d y \\
& +\int_{\Omega} A(t) v^{m} \Delta v_{t}^{m} d y+\int_{\Omega} a_{1} \cdot \nabla v_{t}^{m} \Delta v_{t}^{m} d y+\int_{\Omega} a_{2} \cdot \nabla v^{m} \Delta v_{t}^{m} d y=0
\end{aligned}
$$

Using Lemma 2.1, we obtain

$$
\begin{aligned}
\int_{\Omega} \int_{0}^{t} g(t-s) \gamma^{-2}(s) \Delta v^{m}(s) \Delta v_{t}^{m} d y & \\
= & -\frac{1}{2} g(t) \gamma^{-2}(0)\left\|\Delta v^{m}\right\|_{L^{2}(\Omega)}^{2}+\frac{1}{2} g^{\prime} \square \frac{\Delta v^{m}}{\gamma} \\
& -\frac{1}{2} \frac{d}{d t}\left[g \square \frac{\Delta v^{m}}{\gamma}-\left(\int_{0}^{t} g(s) \gamma^{-2}(s) d s\right)\left\|\Delta v^{m}\right\|_{L^{2}(\Omega)}^{2}\right] .
\end{aligned}
$$


M. L. Santos et al. 909

Substituting (2.17) into (2.16), we get

$$
\begin{aligned}
\frac{1}{2} \frac{d}{d t} \mathfrak{L}_{2}^{m}( & t)+\alpha\left\|\nabla v_{t}^{m}\right\|_{L^{2}(\Omega)}^{2} \\
= & -\frac{1}{2} \frac{g(t)}{\gamma^{2}(0)}\left\|\Delta v^{m}\right\|_{L^{2}(\Omega)}^{2}+\frac{1}{2} g^{\prime} \square \frac{\Delta v^{m}}{\gamma}-\frac{2 \gamma^{\prime}}{\gamma^{5}}\left\|\nabla \Delta v^{m}\right\|_{L^{2}(\Omega)}^{2} \\
& +\left(A(t) v^{m}, \Delta v_{t}^{m}\right)+\left(a_{1} \cdot \nabla v_{t}^{m}, \Delta v_{t}^{m}\right)+\left(a_{2} \cdot \nabla v^{m}, \Delta v_{t}^{m}\right) \\
& +\frac{1}{2} \frac{d}{d t}\left(\gamma^{-2} M\left(\gamma^{n-2}\left\|\nabla v^{m}\right\|_{L^{2}(\Omega)}^{2}\right)\right)\left\|\Delta v^{m}\right\|_{L^{2}(\Omega)}^{2},
\end{aligned}
$$

where

$$
\begin{aligned}
\mathfrak{L}_{2}^{m}(t)= & \left\|\nabla v_{t}^{m}\right\|_{L^{2}(\Omega)}^{2}+g \square \frac{\Delta v^{m}}{\gamma}-\left(\int_{0}^{t} g(s) \gamma^{-2}(s) d s\right)\left\|\Delta v^{m}\right\|_{L^{2}(\Omega)}^{2} \\
& +\gamma^{-4}\left\|\nabla \Delta v^{m}\right\|_{L^{2}(\Omega)}^{2}+\gamma^{-2} M\left(\gamma^{n-2}\left\|\nabla v^{m}\right\|_{L^{2}(\Omega)}^{2}\right)\left\|\Delta v^{m}\right\|_{L^{2}(\Omega)}^{2} .
\end{aligned}
$$

From (1.27), (1.28), and (1.30), we have

$$
\gamma^{-4}\left\|\nabla \Delta v^{m}\right\|_{L^{2}(\Omega)}^{2}+\gamma^{-2} M\left(\gamma^{n-2}\left\|\nabla v^{m}\right\|_{L^{2}(\Omega)}^{2}\right)\left\|\Delta v^{m}\right\|_{L^{2}(\Omega)}^{2} \geq \frac{m_{1}}{\|\gamma\|_{L^{\infty}}^{2}}\left\|\Delta v^{m}\right\|_{L^{2}(\Omega)}^{2} .
$$

Using relation (2.18) and taking into account (2.20), we get

$$
\mathfrak{E}_{2}^{m}(t)+\alpha \int_{0}^{t}\left\|\nabla v_{s}^{m}(s)\right\|_{L^{2}(\Omega)}^{2} d s \leq C_{1}+C_{2} \int_{0}^{t}\left(\left|\gamma^{\prime}\right|+\left|\gamma^{\prime \prime}\right|\right) \mathfrak{E}_{2}^{m}(s) d s .
$$

Using Gronwall's and taking into account (1.24), we get

$$
\mathfrak{E}_{2}^{m}(t)+\alpha \int_{0}^{t}\left\|\nabla v_{s}^{m}(s)\right\|_{L^{2}(\Omega)}^{2} d s \leq C \quad \forall t \in[0, T], \forall m \in \mathbb{N} .
$$

A priori estimate III. Differentiating (2.9) with respect to the time, multiplying by $g_{j m}^{\prime \prime}(t)$, and using similar arguments as (2.22), we obtain, after some calculations and taking into account (2.22),

$$
\begin{aligned}
\frac{1}{2} \frac{d}{d t} \mathfrak{L}_{3}^{m}(t)+\alpha\left\|v_{t t}^{m}(t)\right\|_{L^{2}(\Omega)}^{2} \leq & C\left(\left|\gamma^{\prime}\right|+\left|\gamma^{\prime \prime}\right|\right)\left\|v_{t}^{m}(t)\right\|_{L^{2}(\Omega)}^{2} \\
& +C\left(\left|\gamma^{\prime}\right|+\left|\gamma^{\prime \prime}\right|\right) \mathfrak{E}_{3}^{m}(t), \quad \forall t \in[0, T], \forall m \in \mathbb{N},
\end{aligned}
$$

where

$$
\begin{aligned}
\mathfrak{E}_{3}^{m}(t)= & \left\|v_{t t}^{m}\right\|_{L^{2}(\Omega)}^{2}+\gamma^{-2} M\left(\gamma^{n-2}\left\|\nabla v^{m}\right\|_{L^{2}(\Omega)}^{2}\right)\left\|\nabla v_{t}^{m}\right\|_{L^{2}(\Omega)}^{2} \\
& +g \square \frac{\nabla v_{t}^{m}}{\gamma}-\left(\int_{0}^{t} g(s) \gamma^{-2}(s) d s\right)\left\|\nabla v_{t}^{m}\right\|_{L^{2}(\Omega)}^{2} .
\end{aligned}
$$

Using Gronwall's lemma and relations (2.15), (2.22), we get

$$
\mathfrak{E}_{3}^{m}(t)+\alpha \int_{0}^{t}\left\|\Delta v^{m}(s)\right\|_{L^{2}(\Omega)}^{2} d s \leq C, \quad \forall t \in[0, T], \forall m \in \mathbb{N} .
$$


910

Uniform decay for a nonlinear beam equation

It easy to see from (2.9) that

$$
\left\|v_{t t}^{m}(0)\right\|_{L^{2}(\Omega)}^{2} \leq C \quad \forall m \in N .
$$

A priori estimate $I V$. Setting $w=v^{m}(t)$ in (2.9), we deduce that

$$
\begin{aligned}
\frac{1}{2} \frac{d}{d t} \mathfrak{E}_{4}^{(m)} & (t)-\left\|v_{t}^{m}\right\|_{L^{2}(\Omega)}^{2}+\gamma^{-4}\left\|\Delta v^{m}\right\|_{L^{2}(\Omega)}^{2} \\
& +\gamma^{-2} M\left(\gamma^{n-2}\left\|\nabla v^{m}\right\|_{L^{2}(\Omega)}^{2}\right)\left\|\nabla v^{m}\right\|_{L^{2}(\Omega)}^{2} \\
& +\int_{0}^{t} g(t-s) \Delta v^{m}(s) v^{m}(s) d s \leq C\left(\left|\gamma^{\prime}\right|+\left|\gamma^{\prime \prime}\right|\right) \\
& \times\left(\left\|v^{m}\right\|_{L^{2}(\Omega)}^{2}+\left\|\Delta v^{m}\right\|_{L^{2}(\Omega)}^{2}+\left\|v_{t}^{m}\right\|_{L^{2}(\Omega)}^{2}\right),
\end{aligned}
$$

where

$$
\mathfrak{E}_{4}^{m}(t)=2 \int_{\Omega} v^{m} v_{t}^{m} d y+\alpha\left\|v^{m}\right\|_{L^{2}(\Omega)}^{2}
$$

From (1.27), (1.28), and (1.30), we have

$$
\gamma^{-4}\left\|\Delta v^{m}\right\|_{L^{2}(\Omega)}^{2}+\gamma^{-n} \hat{M}\left(\gamma^{n-2}\left\|\nabla v^{m}\right\|_{L^{2}(\Omega)}^{2}\right) \geq \frac{m_{1}}{\|\gamma\|_{L^{\infty}}^{2}}\left\|\nabla v^{m}\right\|_{L^{2}(\Omega)}^{2},
$$

where $m_{1}=\left(\lambda_{1} /\|\gamma\|_{L^{\infty}}^{2}-m_{0}\right)>0$. Moreover, it is easy to see that choosing $k>2 / \alpha$ (see also (2.29)), we obtain

$$
\begin{aligned}
k \mathfrak{E}_{1}^{m}(t)+\mathfrak{E}_{4}^{m}(t) \geq & \left(k-\frac{2}{\alpha}\right)\left(\left\|v_{t}^{m}\right\|_{L^{2}(\Omega)}^{2}+\left\|v^{m}\right\|_{L^{2}(\Omega)}^{2}\right) \\
& +\left(k-\frac{2}{\alpha}\right)\left(\gamma^{-4}\left\|\Delta v^{m}\right\|_{L^{2}(\Omega)}^{2}+\gamma^{-n} \hat{M}\left(\gamma^{n}\left\|\nabla v^{m}\right\|_{L^{2}(\Omega)}^{2}\right)\right)>0 .
\end{aligned}
$$

Now, multiplying (2.11) by $k$ and combining with (2.27), we get, taking into account (2.29),

$$
\begin{aligned}
& \frac{1}{2} \frac{d}{d t}\left(k \mathfrak{E}_{1}^{m}(t)+\mathfrak{E}_{4}^{m}(t)\right)+(k \alpha-1)\left\|v_{t}^{m}\right\|_{L^{2}(\Omega)}^{2}+\gamma^{-4}\left\|\Delta v^{m}\right\|_{L^{2}(\Omega)}^{2} \\
& \quad \leq C\left(\left|\gamma^{\prime}\right|+\left|\gamma^{\prime \prime}\right|\right)\left(k \mathfrak{E}_{1}^{m}(t)+\mathfrak{E}_{4}^{m}(t)\right) .
\end{aligned}
$$

From (2.31), using Gronwall's lemma, we obtain the following estimate:

$$
\begin{aligned}
k \mathfrak{E}_{1}^{n}(t) & +\mathfrak{E}_{4}^{m}(t)+\int_{0}^{t}\left(\left\|v_{t}^{m}\right\|_{L^{2}(\Omega)}^{2}+\left\|\Delta v^{m}\right\|_{L^{2}(\Omega)}^{2}\right) d s \\
\leq & C\left(\left\|v_{1}\right\|_{L^{2}(\Omega)}^{2}+\left\|\Delta v_{0}\right\|_{L^{2}(\Omega)}^{2}\right) \exp \left(C \int_{0}^{\infty}\left(\gamma^{\prime}(t)+\gamma^{\prime \prime}(t)\right) d t\right) .
\end{aligned}
$$


In virtue of (2.29) and (1.26), it follows from (2.32) that

$$
\begin{gathered}
\left\|v_{t}^{m}\right\|_{L^{2}(\Omega)}^{2}+\left\|\Delta v^{m}\right\|_{L^{2}(\Omega)}^{2}+\int_{0}^{t}\left(\left\|v_{t}^{m}\right\|_{L^{2}(\Omega)}^{2}+\left\|\Delta v^{m}\right\|_{L^{2}(\Omega)}^{2}\right) d s \\
\leq C\left(\left\|v_{1}\right\|_{L^{2}(\Omega)}^{2}+\left\|\Delta v_{0}\right\|_{L^{2}(\Omega)}^{2}\right) .
\end{gathered}
$$

From estimates (2.15), (2.22), (2.25), and (2.33), it follows that $v^{m}$ converges strongly in $L^{2}\left(0, \infty: H^{1}(\Omega)\right)$ to some $v \in L^{2}\left(0, \infty: H^{1}(\Omega)\right)$. Moreover, since $M \in C^{1}[0, \infty)$ and $\nabla v^{m}$ is bounded in $L^{\infty}\left(0, \infty: L^{2}(\Omega)\right) \cap L^{2}\left(0, \infty: L^{2}(\Omega)\right)$, we have

$$
\int_{0}^{t}\left|M\left(\gamma^{n-2}\left\|\nabla v^{m}\right\|_{L^{2}(\Omega)}^{2}\right)-M\left(\gamma^{n-2}\|\nabla v\|_{L^{2}(\Omega)}^{2}\right)\right| d s \leq C \int_{0}^{t}\left\|v^{m}-v\right\|_{H^{1}(\Omega)}^{2} d s,
$$

where $C$ is a positive constant independent of $m$ and $t$ so that

$$
M\left(\gamma^{n-2}\left\|\nabla v^{m}\right\|_{L^{2}(\Omega)}^{2}\right)\left(\Delta v^{m}, w_{j}\right) \longrightarrow M\left(\gamma^{n-2}\|\nabla v\|_{L^{2}(\Omega)}^{2}\right)\left(\Delta v, w_{j}\right) .
$$

Therefore, we have that $v$ satisfies

$$
\begin{gathered}
v \in L^{\infty}\left(0, \infty: H_{0}^{1}(\Omega)\right) \cap L^{2}\left(0, \infty: H_{0}^{2}(\Omega)\right), \\
v_{t} \in L^{\infty}\left(0, \infty: H_{0}^{1}(\Omega)\right), \\
v_{t t} \in L^{\infty}\left(0, \infty: L^{2}(\Omega)\right) .
\end{gathered}
$$

Letting $m \rightarrow \infty$ in (2.9), we conclude that

$$
\begin{aligned}
v_{t t} & +\gamma^{-4} \Delta^{2} v-\gamma^{-2} M\left(\gamma^{n-2}\|\nabla v\|_{L^{2}(\Omega)}^{2}\right) \Delta v+\int_{0}^{t} g(t-s) \gamma^{-2}(s) \Delta v(s) d s \\
& +\alpha v_{t}-A(t) v+a_{1} \cdot \nabla \partial_{t} v+a_{2} \cdot \nabla v=0
\end{aligned}
$$

in $L^{\infty}\left(0, \infty: L^{2}(\Omega)\right)$. Therefore, we have

$$
v \in L^{\infty}\left(0, \infty: H_{0}^{2}(\Omega) \cap H^{4}(\Omega)\right) .
$$

To prove the uniqueness of solutions of the problem (1.21), we use the method of the energy introduced by Lions [16], coupled with Gronwall's inequality and the hypotheses introduced in the paper about the functions $M, g$, and the obtained estimates.

To show the existence in noncylindrical domains, we return to our original problem in the noncylindrical domains by using the change variable given in (1.18) by $(y, t)=\tau(x, t)$, $(x, t) \in \hat{Q}$. Let $v$ be the solution obtained from Theorem 2.2 and $u$ defined by (1.20), then $u$ belongs to the classes

$$
\begin{gathered}
u \in L^{\infty}\left(0, \infty: H_{0}^{2}\left(\Omega_{t}\right) \cap H^{4}\left(\Omega_{t}\right)\right), \\
u_{t} \in L^{\infty}\left(0, \infty: H_{0}^{1}\left(\Omega_{t}\right)\right), \\
u_{t t} \in L^{\infty}\left(0, \infty: L^{2}\left(\Omega_{t}\right)\right) .
\end{gathered}
$$


912 Uniform decay for a nonlinear beam equation

Denoting that

$$
u(x, t)=v(y, t)=(v \circ \tau)(x, t)
$$

then from (1.20) follows that

$$
u_{t t}+\Delta^{2} u-M\left(\|\nabla u\|_{L^{2}\left(\Omega_{t}\right)}^{2}\right) \Delta u+\int_{0}^{t} g(t-s) \Delta u(s) d s+\alpha u_{t}=0
$$

in $L^{\infty}\left(0, \infty: L^{2}\left(\Omega_{t}\right)\right)$. If $u_{1}, u_{2}$ are two solutions obtained through the diffeomorphism $\tau$ given by (1.18), then $v_{1}, v_{2}$ are the solutions to (1.20). By uniqueness result of Theorem 2.2, we have $v_{1}=v_{2}$, so $u_{1}=u_{2}$. Therefore, we have the following result.

Theorem 2.3. Take $u_{0} \in H_{0}^{2}\left(\Omega_{0}\right) \cap H^{4}\left(\Omega_{0}\right), u_{1} \in H_{0}^{2}\left(\Omega_{0}\right)$, and suppose that assumptions (1.24), (1.25), (1.26), (1.27), (1.28), (1.30), and (2.1) hold. Then there exists a unique solution $u$ of the problem (1.11)-(1.13) satisfying (2.39) and the equation

$$
u_{t t}+\Delta^{2} u-M\left(\|\nabla u\|_{L^{2}\left(\Omega_{t}\right)}^{2}\right) \Delta u+\int_{0}^{t} g(t-s) \Delta u(s) d s+\alpha u_{t}=0
$$

in $L^{\infty}\left(0, \infty: L^{2}\left(\Omega_{t}\right)\right)$.

\section{Exponential decay}

In this section, we show that the solution of system (1.11)-(1.13) decays exponentially. To this end, we will assume that the memory $g$ satisfies

$$
\begin{gathered}
g^{\prime}(t) \leq-C_{1} g(t), \\
\left(m_{0}-\int_{0}^{\infty} g(s) d s\right)=\beta_{1}>0,
\end{gathered}
$$

for all $t \geq 0$ with positive constants $C_{1}$. Additionally, we assume that the function $\gamma(\cdot)$ satisfies the conditions

$$
\begin{gathered}
\gamma^{\prime} \leq 0, \quad t \geq 0, \quad n>2, \\
0<\max _{0 \leq t<\infty}\left|\gamma^{\prime}(t)\right| \leq \frac{1}{d},
\end{gathered}
$$

where $d=\operatorname{diam}(\Omega)$. The condition (3.4) (see also (1.17)) implies that our domain are "time-like" in the sense that

$$
|\underline{\nu}|<|\bar{\nu}|
$$

where $\underline{v}$ and $\bar{v}$ denote the $t$-component and $x$-component of the outer unit normal of $\hat{\sum}$. 
Remark 3.1. It is important to observe that to prove the main theorem of this section, that is, Theorem 3.8 as well as Lemma 3.6, we use the following substantial hypothesis:

$$
M(s) \geq m_{0}>0, \quad \forall s \in[0, \infty[.
$$

This is because we worked directly in our domain with moving boundary, where the geometry of our domain influences directly the problem, which generated several technical difficulties in limiting some terms in Lemma 3.6, and consequently in proving Theorem 3.8. To facilitate our calculations, we introduce the following notation:

$$
(g \square \nabla u)(t)=\int_{\Omega_{t}} \int_{0}^{t} g(t-s)|\nabla u(t)-\nabla u(s)|^{2} d s d x .
$$

First of all, we will prove the following three lemmas that will be used in the sequel. Lemma 3.2. Let $F(\cdot, \cdot)$ be the smooth function defined in $\Omega_{t} \times[0, \infty[$. Then,

$$
\frac{d}{d t} \int_{\Omega_{t}} F(x, t) d x=\int_{\Omega_{t}} \frac{d}{d t} F(x, t) d x+\frac{\gamma^{\prime}}{\gamma} \int_{\Gamma_{t}} F(x, t)(x \cdot \bar{v}) d \Gamma_{t},
$$

where $\bar{\nu}$ is the $x$-component of the unit normal exterior $\nu$.

For the proof, see for example [7].

Lemma 3.3. Let $v \in H^{2}(\Omega) \cap H_{0}^{1}(\Omega)$. Then for all $i=1, \ldots, n$,

$$
\frac{\partial v}{\partial y_{i}}=\eta_{i} \frac{\partial v}{\partial \nu}
$$

Proof. We consider $r \in C^{2}\left(\bar{\Omega}, \mathbb{R}^{n}\right)$ such that

$$
r=v \quad \text { on } \Gamma \text {. }
$$

(It is possible to choose such a field $r(\cdot)$ if we consider that the boundary $\Gamma$ is sufficiently smooth.) Let $\theta \in \mathscr{D}(\Gamma)$ and $\varphi \in H^{m}(\Omega)$ with $m>\max (n / 2,2)$ such that $\left.\varphi\right|_{\Gamma}=\theta$. Since $\mathscr{D}(\Gamma) \subset H^{m-1 / 2}(\Gamma)$, such function $\varphi$ exists and we have

$$
\int_{\Omega} \frac{\partial^{2}}{\partial y_{i} \partial y_{j}}\left(v r_{j} \varphi\right) d y=\int_{\Gamma} v_{j} \frac{\partial}{\partial y_{j}}\left(v r_{j} \varphi\right) d \Gamma=\int_{\Gamma} \theta v_{i} \frac{\partial v}{\partial \nu} d \Gamma \quad(i, j=1, \ldots, n) .
$$

Note that $\Omega$ is regular, we also obtain

$$
\int_{\Omega} \frac{\partial^{2}}{\partial y_{j} \partial y_{i}}\left(v r_{j} \varphi\right) d y=\int_{\Gamma} v_{j} \frac{\partial}{\partial y_{i}}\left(v r_{j} \varphi\right) d \Gamma=\int_{\Gamma} \theta v_{j}^{2} \frac{\partial v}{\partial y_{i}} d \Gamma=\int_{\Gamma} \theta \frac{\partial v}{\partial y_{i}} d \Gamma .
$$

It follows that

$$
\int_{\Gamma} \theta \frac{\partial v}{\partial y_{i}} d \Gamma=\int_{\Gamma} \theta\left(v_{i} \frac{\partial v}{\partial y_{i}}\right) d \Gamma \quad \forall \theta \in \mathscr{D}(\Gamma)
$$

which implies (3.9). 
914 Uniform decay for a nonlinear beam equation

From Lemma 3.3, it is easy to see that

$$
\nabla u \cdot \bar{v}=\frac{\partial u}{\partial \bar{v}} \quad \text { on } \Gamma_{t}
$$

and for $u \in H_{0}^{2}\left(\Omega_{t}\right) \cap H^{4}\left(\Omega_{t}\right)$ (see Komornik [10, page 26]), we have

$$
|\nabla u|=0, \quad \frac{\partial^{2} u}{\partial \bar{v}^{2}}=\Delta u-\frac{\partial u}{\partial \bar{\nu}} \operatorname{div} \bar{\nu}=\Delta u \quad \text { on } \Gamma_{t} .
$$

Lemma 3.4. For any function $g \in C^{1}\left(\mathbb{R}_{+}\right)$and $u \in C^{1}\left(0, \infty: H_{0}^{2}\left(\Omega_{t}\right) \cap H^{4}\left(\Omega_{t}\right)\right)$,

$$
\begin{aligned}
\int_{\Omega_{t}} \int_{0}^{t} g(t-s) \nabla u(s) \cdot \nabla u_{t}(t) d s d x \\
=-\frac{1}{2} g(t) \int_{\Omega_{t}}|\nabla u(t)|^{2} d x+\frac{1}{2} g^{\prime} \square \nabla u \\
\quad-\frac{1}{2} \frac{d}{d t}\left[g \square \nabla u-\left(\int_{0}^{t} g(s) d s\right) \int_{\Omega_{t}}|\nabla u|^{2}\right] .
\end{aligned}
$$

Proof. Differentiating the term $g \square \nabla u$ and applying Lemma 3.2, we obtain

$$
\begin{aligned}
\frac{d}{d t} g \square \nabla u= & \int_{\Omega_{t}} \frac{d}{d t} \int_{0}^{t} g(t-s)|\nabla u(t)-\nabla u(s)|^{2} d s d x \\
& +\frac{\gamma^{\prime}}{\gamma} \int_{\Gamma_{t}} \int_{0}^{t} g(t-s)|\nabla u(t)-\nabla u(s)|^{2}(x \cdot \bar{\nu}) d s d \Gamma_{t} .
\end{aligned}
$$

Using (3.15), we have

$$
\begin{aligned}
\frac{d}{d t} g \square \nabla u= & \int_{\Omega_{t}} \int_{0}^{t} g^{\prime}(t-s)|\nabla u(t)-\nabla u(s)|^{2} d s d x \\
& -2 \int_{\Omega_{t}} \int_{0}^{t} g(t-s) \nabla u_{t}(t) \cdot \nabla u(s) d s d x \\
& +\left(\int_{0}^{t} g(t-s) d s\right) \int_{\Omega_{t}} \frac{d}{d t}|\nabla u(t)|^{2} d x
\end{aligned}
$$

from where it follows that

$$
\begin{aligned}
2 \int_{\Omega_{t}} \int_{0}^{t} g(t-s) \nabla u_{t}(t) \cdot \nabla u(s) d s d x \\
=-\frac{d}{d t}\left\{g \square \nabla u-\int_{0}^{t} g(t-s) d s \int_{\Omega_{t}}|\nabla u(t)|^{2} d x\right\} \\
\quad+\int_{\Omega_{t}} \int_{0}^{t} g^{\prime}(t-s)|\nabla u(t)-\nabla u(s)|^{2} d s d x-g(t) \int_{\Omega_{t}}|\nabla u(t)|^{2} d x
\end{aligned}
$$

The proof is now complete. 
M. L. Santos et al. 915

We introduce the functional

$$
\begin{aligned}
E(t)= & \left\|u_{t}\right\|_{L^{2}\left(\Omega_{t}\right)}^{2}+\|\Delta u\|_{L^{2}\left(\Omega_{t}\right)}^{2}+\hat{M}\left(\|\nabla u\|_{L^{2}\left(\Omega_{t}\right)}^{2}\right) \\
& -\left(\int_{0}^{t} g(s) d s\right)\|\nabla u\|_{L^{2}\left(\Omega_{t}\right)}^{2}+g \square \nabla u .
\end{aligned}
$$

We observe that $E(t)>0$ since the hypotheses (2.1), (3.2), and (3.6) are satisfied.

LEMmA 3.5. Take $u_{0} \in H_{0}^{2}\left(\Omega_{0}\right) \cap H^{4}\left(\Omega_{0}\right), u_{1} \in H_{0}^{2}\left(\Omega_{0}\right)$, and suppose that assumptions (1.24), (1.25), (1.26), (1.27), (1.28), (1.30), (2.1), and (3.6) hold. Then there exists a unique strong global solution of (1.11)-(1.13) satisfying

$$
\frac{d}{d t} E(t)+2 \alpha\left\|u_{t}\right\|_{L^{2}\left(\Omega_{t}\right)}^{2} \leq-\int_{\Omega_{t}} g(t)|\nabla u|^{2} d x+g^{\prime} \square \nabla u \text {. }
$$

Proof. Multiplying (1.11) by $u_{t}$, performing an integration by parts over $\Omega_{t}$, and using Lemma 3.2, we obtain

$$
\begin{aligned}
\frac{1}{2} \frac{d}{d t}\left\|u_{t}\right\|_{L^{2}\left(\Omega_{t}\right)}^{2}+\frac{1}{2} \frac{d}{d t}\|\nabla u\|_{L^{2}\left(\Omega_{t}\right)}^{2}+\frac{1}{2} \frac{d}{d t}\|\Delta u\|_{L^{2}\left(\Omega_{t}\right)}^{2} & \\
+ & \frac{1}{2} \frac{d}{d t} \hat{M}\left(\|\nabla u\|_{L^{2}\left(\Omega_{t}\right)}^{2}\right)+\alpha\left\|u_{t}\right\|_{L^{2}\left(\Omega_{t}\right)}^{2}-\int_{\Omega_{t}} \int_{0}^{t} g(t-s) \nabla u(s) \cdot \nabla u_{t} d s d x \\
& -\frac{\gamma^{\prime}}{2 \gamma} M\left(\|\nabla u\|_{L^{2}\left(\Omega_{t}\right)}^{2}\right) \int_{\Gamma_{t}}|\nabla u|^{2}(\bar{\nu} \cdot x)-\int_{\Gamma_{t}} \frac{\gamma^{\prime}}{2 \gamma}(\bar{\nu} \cdot x)\left(\left|u_{t}\right|^{2}+|\Delta u|^{2}\right) d \Gamma_{t}=0 .
\end{aligned}
$$

Using (3.3), we obtain

$$
-\frac{\gamma^{\prime}}{2 \gamma} M\left(\|\nabla u\|_{L^{2}\left(\Omega_{t}\right)}^{2}\right) \int_{\Gamma_{t}}|\nabla u|^{2}(\bar{\nu} \cdot x)-\int_{\Gamma_{t}} \frac{\gamma^{\prime}}{2 \gamma}(\bar{\nu} \cdot x)\left(\left|u_{t}\right|^{2}+|\Delta u|^{2}\right) d \Gamma_{t} \geq 0 .
$$

Taking into account the above inequality and (3.14) and Lemma 3.4, we obtain the conclusion of the lemma.

We consider the following functional:

$$
\psi(t)=2 \int_{\Omega_{t}} u_{t} u d x+\alpha\|u\|_{L^{2}\left(\Omega_{t}\right)}^{2} .
$$

LemmA 3.6. Take $u_{0} \in H_{0}^{2}\left(\Omega_{0}\right) \cap H^{4}\left(\Omega_{0}\right), u_{1} \in H_{0}^{2}\left(\Omega_{0}\right)$, and suppose that assumptions (1.24), (1.25), (1.26), (1.27), (1.28), (1.30), (2.1), and (3.6) hold. Then there exists a unique strong global solution of (1.11)-(1.13) satisfying

$$
\begin{aligned}
\frac{1}{2} \frac{d}{d t} \psi(t) \leq & \left\|u_{t}\right\|_{L^{2}\left(\Omega_{t}\right)}^{2}-M\left(\|\nabla u\|_{L^{2}\left(\Omega_{t}\right)}^{2}\right)\|\nabla u\|_{L^{2}\left(\Omega_{t}\right)}^{2}+\left(\int_{0}^{t} g(s) d s\right)\|\nabla u\|_{L^{2}\left(\Omega_{t}\right)}^{2} \\
& -\|\Delta u\|_{L^{2}\left(\Omega_{t}\right)}^{2}+\|\nabla u\|_{L^{2}\left(\Omega_{t}\right)}\left(\int_{0}^{t} g(s) d s\right)^{1 / 2}(g \square \nabla u)^{1 / 2} .
\end{aligned}
$$


916 Uniform decay for a nonlinear beam equation

Proof. Multiplying (1.11) by $u$ and integrating over $\Omega_{t}$, we obtain

$$
\begin{aligned}
\frac{1}{2} \frac{d}{d t} \psi(t)= & \left\|u_{t}\right\|_{L^{2}\left(\Omega_{t}\right)}^{2}-\|\Delta u\|_{L^{2}\left(\Omega_{t}\right)}^{2}-M\left(\|\nabla u\|_{L^{2}\left(\Omega_{t}\right)}^{2}\right)\|\nabla u\|_{L^{2}\left(\Omega_{t}\right)}^{2} \\
& +\int_{\Omega_{t}} \int_{0}^{t} g(t-s) \nabla u(s) \cdot \nabla u d s d x
\end{aligned}
$$

Noting that

$$
\begin{aligned}
\int_{\Omega_{t}} \int_{0}^{t} g(t-s) \nabla u(s) \cdot \nabla u d s d x= & \int_{\Omega_{t}} \int_{0}^{t} g(t-s)(\nabla u(s)-\nabla u(t)) \cdot \nabla u d s d x \\
& +\int_{\Omega_{t}}\left(\int_{0}^{t} g(s) d s\right)|\nabla u|^{2} d x,
\end{aligned}
$$

and taking into account that

$$
\left|\int_{\Omega_{t}} \int_{0}^{t} g(t-s)(\nabla u(s)-\nabla u(t)) \cdot \nabla u d s d x\right| \leq\|\nabla u\|_{L^{2}\left(\Omega_{t}\right)}\left(\int_{0}^{t} g(s) d s\right)^{1 / 2}(g \square \nabla u)^{1 / 2},
$$

there follows the conclusion of lemma.

Remark 3.7. We used the hypotheses (1.24), (1.25), (1.26), (1.27), (1.28), (1.30), (2.1), and (3.6) because we are interested in strong global solution for our problem (1.11)(1.13), which was obtained by the existence and uniqueness in Section 2.

We introduce the functional

$$
\mathscr{L}(t)=N E(t)+\psi(t)
$$

with $N>0$. It is not difficult to see that $\mathscr{L}(t)$ verifies

$$
k_{0} E(t) \leq \mathscr{L}(t) \leq k_{1} E(t)
$$

for $k_{0}$ and $k_{1}$ positive constants. Now we are in a position to show the main result of this paper.

Theorem 3.8. Take $u_{0} \in H_{0}^{2}\left(\Omega_{0}\right) \cap H^{4}\left(\Omega_{0}\right), u_{1} \in H_{0}^{2}\left(\Omega_{0}\right)$, and suppose that assumptions (1.24), (1.25), (1.26), (1.27), (1.28), (1.30), (2.1), (3.1), (3.2), (3.4), and (3.6) hold. Also, suppose that $g \in W^{1,2}(0, \infty)$. Then the strong solution of the system (1.11)-(1.13) satisfies

$$
E(t) \leq C e^{-\xi t} E(0), \quad \forall t \geq 0,
$$

where $C$ and $\xi$ are positive constants. 
Proof. Using Lemmas 3.5 and 3.6, we get

$$
\begin{aligned}
\frac{d}{d t} \mathscr{L}(t) \leq & -2 N \alpha\left\|u_{t}\right\|_{L^{2}\left(\Omega_{t}\right)}^{2}-C_{1} N g \square \nabla u+\left\|u_{t}\right\|_{L^{2}\left(\Omega_{t}\right)}^{2} \\
& -\|\Delta u\|_{L^{2}\left(\Omega_{t}\right)}^{2}-\widehat{M}\left(\|\nabla u\|_{L^{2}\left(\Omega_{t}\right)}^{2}\right)+\left(\int_{0}^{t} g(s) d s\right)\|\nabla u\|_{L^{2}\left(\Omega_{t}\right)}^{2} \\
& +\|\nabla u\|_{L^{2}\left(\Omega_{t}\right)}^{2}\left(\int_{0}^{t} g(s) d s\right)^{1 / 2}(g \square \nabla u)^{1 / 2} .
\end{aligned}
$$

Using (3.1) and Young inequality, we obtain for $\epsilon>0$ that

$$
\begin{aligned}
\frac{d}{d t} \mathscr{L}(t) \leq & -2 N \alpha\left\|u_{t}\right\|_{L^{2}\left(\Omega_{t}\right)}^{2}-C_{1} N g \square \nabla u+\left\|u_{t}\right\|_{L^{2}\left(\Omega_{t}\right)}^{2} \\
& -\|\Delta u\|_{L^{2}\left(\Omega_{t}\right)}^{2}-\widehat{M}\left(\|\nabla u\|_{L^{2}\left(\Omega_{t}\right)}^{2}\right)+\left(\int_{0}^{t} g(s) d s\right)\|\nabla u\|_{L^{2}\left(\Omega_{t}\right)}^{2} \\
& +\frac{\epsilon}{2}\|\nabla u\|_{L^{2}\left(\Omega_{t}\right)}^{2}+\frac{\|g\|_{L^{1}(0, \infty)}}{2 \epsilon} g \square \nabla u .
\end{aligned}
$$

Choosing $N$ large enough, $\epsilon$ small, and using hypothesis (3.6), we obtain

$$
\frac{d}{d t} \mathscr{L}(t) \leq-\lambda_{0} E(t)
$$

where $\lambda_{0}$ is a positive constant independent of $t$. From (3.30) and (3.34), it follows that

$$
\mathscr{L}(t) \leq \mathscr{L}(0) e^{-\left(\lambda_{0} / k_{1}\right) t}, \quad \forall t \geq 0 .
$$

From equivalence relation (3.30) our conclusion follows. The proof now is completed.

Remark 3.9. The techniques in this paper may be used to study the problem (1.11)(1.13) without the term weak dissipative $\alpha u_{t}$. In this case, we define other appropriate functionals to prove the exponential and polynomial decay rates of the energy of regular solutions for the nonlinear beam equations with memory

$$
u_{t t}+\Delta^{2} u-M\left(\|\nabla u\|_{2}^{2}\right) \Delta u+\int_{0}^{t} g(t-s) \Delta u(s) d s=0 \quad \text { in } \hat{Q},
$$

where the functions $M, g$, and $\gamma$ satisfy some appropriate conditions. Results concerning the above equations in domains with moving boundary will appear in a forthcoming paper.

\section{Acknowledgments}

This research was started while the second author was visiting the Federal University of Pará (UFPA)(Pará, Brazil), during March 2003 and the Federal University of São João del Rei (UFSJ)(Minas Gerais, Brazil) during July 2003. He was partially supported by CNPqBrasília under Grant no. 301025/2003-7. The authors are thankful to the referee of this paper for valuable suggestions which improved this paper. Also, the authors would like to 
thank Professor Dr. Djairo G. de Figueiredo for his valuable attention to our paper. The authors would like to express their gratitude to Professor Dr. Jaime E. Muñoz Rivera for the fruitful discussions concerning this paper.

\section{References}

[1] R. Benabidallah and J. Ferreira, Asymptotic behaviour for the nonlinear beam equation in noncylindrical domains, Commun. Appl. Anal. 6 (2002), no. 2, 219-234.

[2] D. Burgreen, Free vibrations of a pinended column with constant distance between pinendes, J. Appl. Mech. 18 (1951), 135-139.

[3] M. M. Cavalcanti, Existence and uniform decay for the Euler-Bernoulli viscoelastic equation with nonlocal boundary dissipation, Discrete Contin. Dyn. Syst. 8 (2002), no. 3, 675-695.

[4] R. Dal Passo and M. Ughi, Problème de Dirichlet pour une classe d'équations paraboliques non linéaires dégénérées dans des ouverts non cylindriques, C. R. Acad. Sci. Paris Sér. I Math. 308 (1989), no. 19, 555-558 (French).

[5] J. G. Eisley, Nonlinear vibration of beams and rectangular plates, Z. Angew. Math. Phys. 15 (1964), 167-175.

[6] J. Ferreira, R. Benabidallah, and J. E. Muñoz Rivera, Asymptotic behaviour for the nonlinear beam equation in a time-dependent domain, Rend. Mat. Appl. (7) 19 (1999), no. 2, 177193.

[7] J. Ferreira and M. L. Santos, Asymptotic behaviour for wave equations with memory in a noncylindrical domains, Commun. Pure Appl. Anal. 2 (2003), no. 4, 511-520.

[8] M. A. Horn, Uniform decay rates for the solutions to the Euler-Bernoulli plate equation with boundary feedback acting via bending moments, Differential Integral Equations 5 (1992), no. $5,1121-1150$.

[9] G. Ji and I. Lasiecka, Nonlinear boundary feedback stabilization for a semilinear Kirchhoff plate with dissipation acting only via moments-limiting behavior, J. Math. Anal. Appl. 229 (1999), no. 2, 452-479.

[10] V. Komornik, Exact Controllability and Stabilization. The Multiplier Method, RAM: Research in Applied Mathematics, Masson, Paris, 1994.

[11] On the nonlinear boundary stabilization of Kirchhoff plates, NoDEA Nonlinear Differential Equations Appl. 1 (1994), no. 4, 323-337.

[12] J. E. Lagnese, Asymptotic energy estimates for Kirch hoff plates subject to weak viscoelastic damping, Control and Estimation of Distributed Parameter Systems (Vorau, 1988), Internat. Ser. Numer. Math., vol. 91, Birkhäuser, Basel, 1989, pp. 211-236.

[13] B Boundary Stabilization of Thin Plates, SIAM Studies in Applied Mathematics, vol. 10, Society for Industrial and Applied Mathematics (SIAM), Pennsylvania, 1989.

[14] I. Lasiecka, Exponential decay rates for the solutions of Euler-Bernoulli equations with boundary dissipation occurring in the moments only, J. Differential Equations 95 (1992), no. 1, 169182.

[15] I. Lasiecka and R. Triggiani, Sharp trace estimates of solutions of Kirchhoff and Euler-Bernoulli equations, Appl. Math. Optim. 28 (1993), no. 3, 277-306.

[16] J.-L. Lions, Quelques Méthodes de Résolution des Problèmes aux Limites non Linéaires, Dunod, Paris, 1969 (French).

[17] J.-L. Lions and E. Magenes, Non-Homogeneous Boundary Value Problems and Applications. Vol. I, Springer-Verlag, New York, 1972.

[18] J. E. Muñoz Rivera, Energy decay rates in linear thermoelasticity, Funkcial. Ekvac. 35 (1992), no. 1, 19-30.

[19] J. E. Muñoz Rivera, E. C. Lapa, and R. Barreto, Decay rates for viscoelastic plates with memory, J. Elasticity 44 (1996), no. 1, 61-87. 
[20] D. C. Pereira, Existence, uniqueness and asymptotic behavior for solutions of the nonlinear beam equation, Nonlinear Anal. 14 (1990), no. 8, 613-623.

[21] O. Ramos Ch., Regularity property for the nonlinear beam operator, An. Acad. Brasil. Ciênc. 61 (1989), no. 1, 15-25.

[22] M. L. Santos and F. Junior, A boundary condition with memory for Kirchhoff plates equations, Appl. Math. Comput. 148 (2004), no. 2, 475-496.

[23] S. Woinowsky-Krieger, The effect of an axial force on the vibration of hinged bars, J. Appl. Mech. 17 (1950), 35-36.

M. L. Santos: Departamento de Matemática, Universidade Federal do Pará, Campus Universitário do Guamá, Rua Augusto Corrêa 01, CEP 66075-110, Pará, Brazil

E-mail address: 1s@ufpa.br

J. Ferreira: Departamento de Matemática, Estatística e Ciências da Computaçäo, Universidade Federal de São João del-Rei (UFSJ), Praça Frei Orlando 170, CEP 36300-000, São João del-Rei, Minas Gerais, Brazil

E-mail address: jf@ufsj.edu.br

C. A. Raposo: Departamento de Matemática, Estatística e Ciências da Computaçäo, Universidade Federal de São João del-Rei (UFSJ), Praça Frei Orlando 170, CEP 36300-000, São João del-Rei, Minas Gerais, Brazil

E-mail address: raposo@ufsj.edu.br 


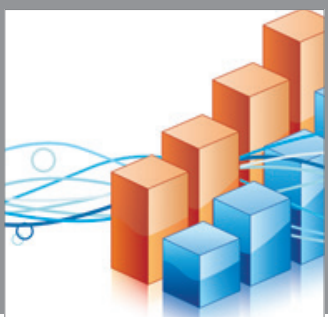

Advances in

Operations Research

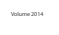

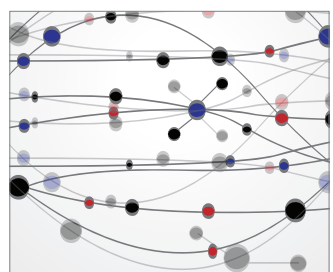

\section{The Scientific} World Journal
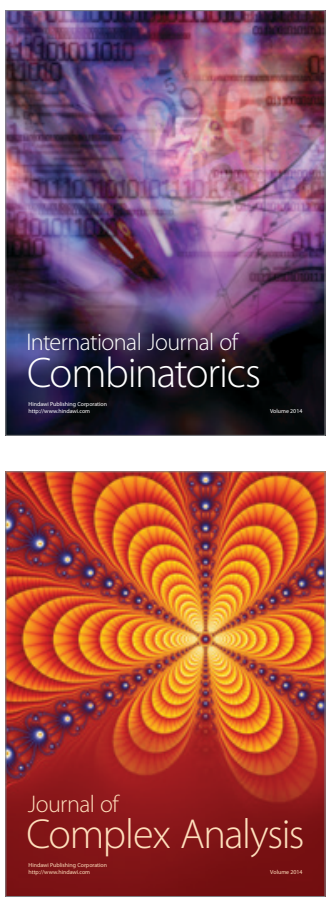

International Journal of

Mathematics and

Mathematical

Sciences
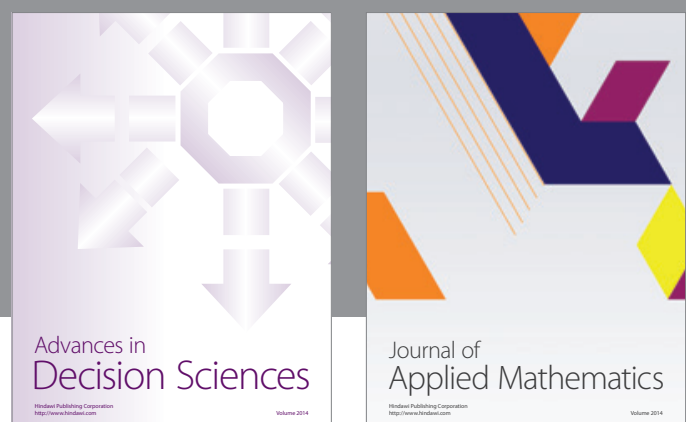

Journal of

Applied Mathematics
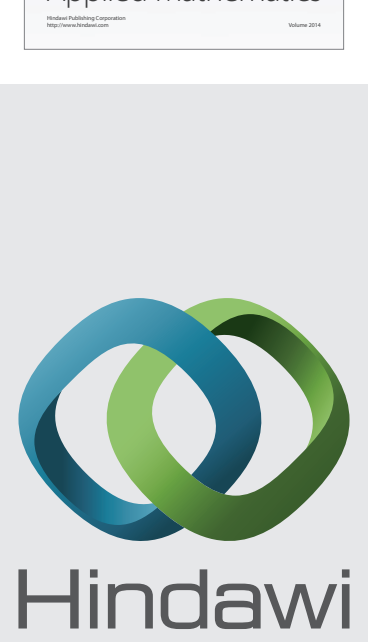

Submit your manuscripts at http://www.hindawi.com
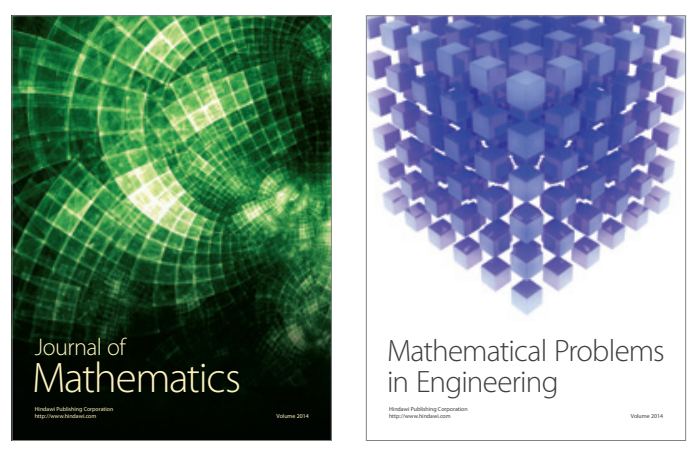

Mathematical Problems in Engineering
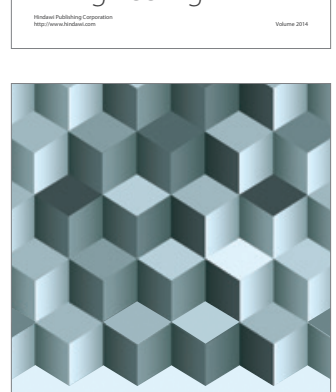

Journal of

Function Spaces
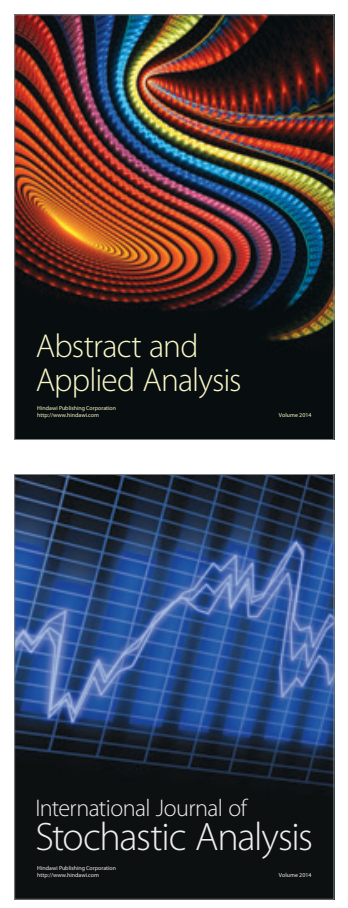

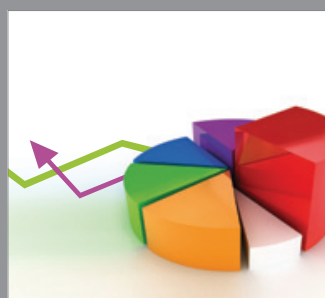

ournal of

Probability and Statistics

Promensencen
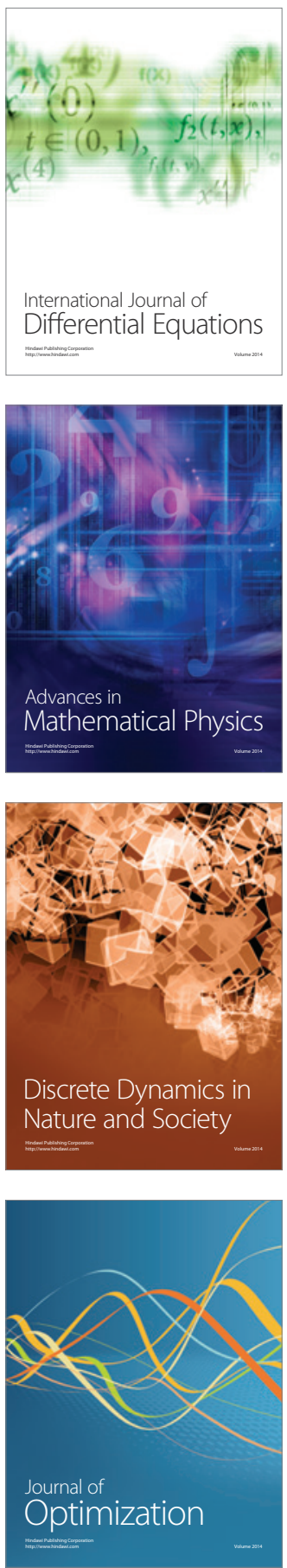